\title{
A TEXT GENERATION SYSTEM FOA EXPLAINING CONCEPTS IN GEOMETRY
}

Ruslan Mitkov

\author{
Institute of mathematice \\ P. O. Box 373, Sofia \\ Butgaria
}

\section{Introduction}

The project note presents the computer program GECO (GEometry consultor), which generates explanations (descriptions) of geometrical concepts in Eulgarian. The program replies to questions such as "What is $x$ ", "Compare $x$ and $V$ ", where $X$ and $Y$ are concepts in geometry. The idea to design GeCO was initially inspired by [Mckeown, 1985], which gives information on missiles. However, unlike TEXT, our systen is able to give two types of descriptions : detailed and brief. For instance, if we ash "What is a triangle", Geco gives a detailed description, including definition, the characteristic and noncharacterietic features of the triangle, but if we ask once again within a dialogue the same question, the program reminds us shortiy the definition of the concept triangle. This is done by means of a special stack, which keeps a formalized dialogue history. As in normal human dialogue, the systems repites to a repeated question in past tense (this is specific at least of Bulgarian). Here is a "recorded" dialogue between user and GECO:

User: What is a triangle?

GECO: The triangle is a convex straightlinear plane geometrical figure, which possesses 3 sides. According to its sides the triangle car be equilateral, isosceles and scalene and according to its angles - rightargled, acuterangled and obtuse-angled.

User: What is a triangle?

GECo: The triangle was a convex,

straightinear and plane geometrical figure. which has three sides.

In our model of natural language generation, we assume, that the task of language generation can be divided into two stages: a conceptual and * linguistic stage. We agree with Danios claim [Dantos, 1985], that the conceptual and inguistic stage cannot be completely independent. That is why not al1 decisions in the conceptual stage of GECO are to be fixed invariabiy.

In [Roesner, 1986] is given a typology of explanations. According to this typology, the explanations, generated by our system are "informative explanations, of static kind" (this type includes the explanations of concepts, what is our case). Roesner proposes, that this type of explanations should possitily include definitions, short descriptive information, indication, where more information can be found, relations betwoen concepts etc. Originally, GECO was intuitively so designed, that it practically met these formal demands.

\section{Sublanguage and discourse}

The system has to de with the sublanguage of school gecmetry (taught in bulgarian 3chools). This sublanguage uses a comparatively restricted lexicon and not too great variety of syntactic constructions. Its texts (what is to be read in the textbooks), however, are often overloaded with very complicated complex sentences. Good school geometry texts are presented in balanced way by simple and complex sentences.

studying discourae pecualiarities of school geonetry instructional texts helped us to design discourse rutes, made use by the conceptuai module, when ordering the content within a text. Generation of text requires the ability how to organize individual sentences. A reasonable writer does not randomily order the sentences in his text, but rather plans an overall framework or outiine, from which the individual sentences are produced. Characteriatic of the description of a geometrical concept is the introduction of its superordinate, its constituents and providing some additional information to it (e.g. varieties). In this way the description of the geometrical concept "quadritateral" possibly includes its superordinate (polygon), itg constituents (4 straightingar sides, which build up a convex figure and 7 ie in one plane, i.d. it has 4 sides and is convex, straightlinear and plane) and its varieties (parallelogram, rectangular etc.).

\section{Sewantic knowledge representiation model}

The semantic know ledge representation mode 1 used in the system and proposed by the autor is an extension of the model of Tiemann and Markle [Tiemann and Mark7e, 1978] for concept semantic 
knowledge representation. The proposed model describes each concept as a set of critical and variable attributes. The concept introduces a class of things, objects, events, ideas or relations, so that each member of this class possesses the same label. On the other hand it is possible that all the members of a class differ in one way or another and nevertheless are classified together. The characteristic features, possessed by all the members in a class are called critical attributes. Variable attributes are defined as characteristic features, which might differ within class members.

Consider the concept "triangle". Our semantic knowledge representation model will describe it formally as follows:

Triangle (geometrical figure/ plane, convex, straightlinear, three sides / acuterangled, right-angled, obtuse-angled: equilateral, isosceles, scalene/0).

\section{The formalis of functional dascriptions}

Different Formalisms require different approaches, whose variety may be sometimes (especialiy in implementation) problematic. We have adopted in our model and system an extended functional description (FD) formalism, developed by Rousselot [Rousseiot, 1985]. This formalism enables the representation of all types of knowledge. A Fo represents a list of attributavalue pairs. Rousselot's formalism is a very extended form of the functional granmars [Kay, 1985]. Within the notation of FD we represent in the domain knowledge base the geometrical concepts (using the above concept semantic representation approach) and the relations among them. We represert also as FDs the grammar rules in the linguistic knowledge base.

\section{The role of logical emphasis}

Different text generation systems make use of different syntax salection appraches. The phenomena of focus is widely used in text generation [Derr and HeKeown, 1984], [McKeown, 1985]. In brief. if the focus is on the protagonist of the sentencs an active construction is chosen, and if the focus is on the goal - a passive one. Thus if the system TEXT [Mckeown,1985] should express the fact, that a triangle and circle touch each other and if the focus is on the protagonist (say triangle), the system would generate "The triangle touches the circle", but if the focus is on the goal (say circie), the following sentence would be produced "The triangle is touched by the circle". Danios [Dan1os,1985] makes use of "discourse graminar", that specifies the syntax of each sentence.
In our work we have elaborated for the first time on the logical emphasis approach for the sentence syntax selection. The logical emphasis in free order Bulgarian is laid on the last word of the sentence. It plays a leading role in choosing the word order in a sentence: the fact that the triangle has three sides may be verbalized as "the triangle has three sides" if the logical emphasis is on "sides" or as "three sides has the triangle" provided the logical emphasis is on "triangle".

\section{Production of text}

The grammar used to produce the preliminary surface structure of the generated sentences is the functiona] unification grammar [Kay, 1985]. If a simple sentence grammar pattern (subject verb object) with valie "protagonist" for the funcional role (attribute) "logical emphasis" is unified with the FD from the domain knowledge base, describing the relation, that each triangle has three sides, the following sentence will be generated:

The triangle powsesses three sides.

while the value "goal" for the attribute "logical emphasis" yields the result:

Three sides has the triangle.

Howevar, the sentence generated by unifying the functional unification grammar with the inout and representing a fact about or rolated to a concept is not the final sentence the system offers to its users. Although the resulting sentences of the previous examples sound quite reasonably (in Bulgarian), the system would not be able to impress always its users if it accepts the sentence as final. The problem is that each explanation of a concept is not a single sentence, but discourse. To iliustrate our position, assume that the system has to give a detailed (initial) description of the concept "triangle" (such description is actually given by our system, see in the dialogue first answer). After consecutive unifications of the grammar rules with the relevant inputs, the system would generate in the best case the following text:

The triangle is a geometrical figure. The triangle is straightlinear. The triangle is plane colane as adjective). The triangle is convex. The triangle has three sides. The triangle can be isosceles, equilateral and scalene according to its sides. The triangle can be right-angled, acute-. angled and obtuse-angles according to its angles. 
This is a clumsy text, that no reasonable man would write. The three main linguistic operations (part of the system's linguistic knowledge) that will process this priliminary text are coordination, pronominalization and ellipsis. Note that coordination will work on the first four sentences, pronominalization - on the fifth sentence and elripsis - on the sentence obtained from coordination of the last two sentences (before these three operations a rhetoric rule will have operated, which says, that "according to" sounds better at the boginning of the sentence and is not subject to deletion during coordination). The processed text will be:

The triangle is a straightlinear. plane and convex geometrical figure, It has three sides. The triangle can be isosceles, equilaterat and scalene according to its sides. The triangle can be right-angled, acute-angled and obtuse-angled according to its angles. According to its sides the triangle can be equilateral, isosceles and scalene and according to its angles - rightangled, acute-angled and obtuse-angled.

There are also further liriguistic decisions to be made: should the sentence be in active or in passive voice, should two or more simpie sentences be combined into a single complex one (in the last sample text the first two sentences can be combined into a complex one), how subordinate clauses should be handled (we have developed severa? procedures to treat the production and connection of subcrdinate clauses), should gerundium be used etc. In text generation systems such decisions are made on the basis of linguistic phenomena such as focus, logical omphasis (in our case), causality etc. and are not to be discussed in the present paper.

\section{Gramatical accordance}

Since Bulgarian is a highly inflective language (inflection affecting not only nouns, but also adjectives, numerals, pronouns etc.), we have developed additional algorithms for grammatical accordance. We have developed an algorithin, which determines automatically the gender of the Bulgarian nouns (consisting of 254 steps). Another algorithm gives the definite article (in Bulgarian as a inflection; there exist various definite article inflections) of each noun and works parallelly to the first one. However, if a Bulgarian noun is in its definite article form, it is impossible to determine algorithmically its gender. Therefore we have daveloped additional algorithms for transforming definite article form of nouns into indefinite article (norma1) forms. Moreover, the adjectives, numera1s, the demonstrative and personal pronouns in Bulgarian accord with the nouns. Consequent iy we have developed and implemented algorithms for determining the indefinite article form of adjectives (numerals, pronouns) and from it the gender form and definite article form of adjectives (numerals, pronouns).

\section{Inp lamentation}

GECO is a program, designed for instructional and experimental purposes. Its most part has been al ready programmed on IBM PC/XT/AT (in Ksi prolog). The fos are described within Prolog Definite Clause crammars (DFG) notation. Thus we have implemented a surface generation based on both the DFG formalism and the formalism of FDs. This idea we have adopted from Derr and Mckeown [Derr and Mckeown, 1984]. The result is a generator with the best features of both grammars: simplification of input by using functional information and efficiency of execution from Prolog.

\section{References}

Danios, Laurence - Generation automatique de textes on langue naturelle, Hasson, Paris, 1985

Derr, Marcia and Mckeown Kathieen - Using focus to generate complex and eimple sentences, COLING, 1984

Kay, Martin - Parsing in functional unification grammar. In Zwickky et al (eds): Natural Tanguage parsing, Cambridge, 1985

Mckeown, Kathieen - Text generation: using discourse strategies and focus contstraints to generate natural language text. Cambridge university press, Cambridge, 1985

Hitkov, Rusian - A knowledge representation mode and its applications, Modess of meaning. Varna, 1988

Roesner, Hannelore - Generierung von Erklaerungen aus formalen Wissensrepraesentation. Bericht No. 3, Verbundvorhaben WISBER, 1986

Rousselot, Francois .. Un systeme comprenant des textes en utilisant ur formalisme unique. T.A. Informations, No.2, 1985

Tiemann P., Markle S - Analyzing instructional content: A guide to instruction and evaluation. Champaign, Il1inois: Stipes publishing company, 1970 